\title{
Evaluación de campo de la precisión de la prueba de diagnóstico rápido SD Bioline Malaria Antigen $\mathrm{Pf} / \mathrm{Pv}{ }^{\circledR}$ en Colombia
}

\author{
Nohora Marcela Mendoza1, Zulma Milena Cucunubá1, Samanda Aponte², \\ Nohora Elizabeth González¹, Sindy Durley Bernal² \\ 1 Grupo de Parasitología, Instituto Nacional de Salud, Bogotá, D.C., Colombia \\ 2 Grupo de Bioquímica, Instituto Nacional de Salud, Bogotá, D.C., Colombia
}

Introducción. Las pruebas de diagnóstico rápido han sido postuladas como una forma de garantizar el diagnóstico de malaria, o paludismo, en zonas de difícil acceso. A pesar de su uso difundido, no hay estudios de campo que evalúen la precisión de la prueba de diagnóstico rápido SD Bioline Malaria Antigen $\mathrm{Pf} / \mathrm{Pv} \AA$ en Colombia.

Objetivo. Evaluar la precisión diagnóstica de la prueba de diagnóstico rápido SD Bioline Malaria Antigen Pf/Pv ${ }^{\circledR}$, en dos departamentos endémicos para malaria, comparando el diagnóstico con la gota gruesa corregida por reacción en cadena de la polimerasa (PCR).

Materiales y métodos. Se trata de un estudio retrospectivo para evaluar sensibilidad, especificidad, valor diagnóstico positivo (VPP) y negativo (VPN), concordancia y límites de sensibilidad por rangos de parasitemia, de la prueba SD Bioline Malaria Antigen ${ }^{\circledR} \mathrm{Pf} / \mathrm{Pv}$, en Córdoba y Chocó. Los resultados fueron comparados con la gota gruesa corregida por PCR.

Resultados. De 383 muestras procesadas, 121 fueron positivas (75 para Plasmodium vivax, 42 para $P$. falciparum y 4 para infección mixta) y 262 muestras negativas; los resultados obtenidos fueron los siguientes: $P$. vivax: sensibilidad, 92,0 \% ( $\mathrm{IC}_{95 \%}$ 83,6-96,3); especificidad, 98,7 \% (IC $\left.{ }_{95 \%} 96,7-99,5\right)$; VPP, 94,5 \% (IC $\left.{ }_{95 \%} 86,7-97,9\right)$; VPN, 98,1\% ( $\left.\mathrm{IC}_{95 \%} 95,8-99,1\right) ; \mathrm{IK}, 0,90$ (0,80-1,00). P. falciparum:

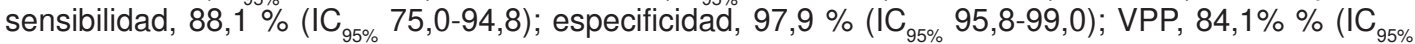
70,6-92,1); VPN, 98,5\% (IC $\left.{ }_{95 \%} 96,6-99,4\right)$; IK, 0,80 (0,70-0,90).

Conclusiones. La prueba tuvo un buen desempeño, siendo mejor para $P$. vivax en comparación con que para $P$. falciparum. Persisten dificultades en la detección de bajas parasitemias.

La falta de amplificación de los genes Pfhrp2 y Pfhrp3 en dos muestras con diagnóstico de como infección mixta, sugiere una posible deleción conjunta de estos genes.

Palabras clave: malaria/diagnóstico, estudios de evaluación, Colombia.

doi: http://dx.doi.org/10.7705/biomedica.v33i4.1464

Field evaluation for diagnostic accuracy of the rapid test SD Bioline Malaria Antigen Pf/Pv® in Colombia

Introduction: Rapid diagnostic tests (RDT) have been postulated as a way to ensure access to malaria diagnosis in remote areas. Despite its widespread use, there are no field studies to evaluate the accuracy of the SD Bioline Malaria Antigen Pf/Pv in Colombia RDT.

Objective: To evaluate the diagnostic accuracy of the SD Bioline Malaria Antigen Pf/Pv® RDT in two departments endemic for malaria, comparing diagnosis with thick film corrected with PCR.

Materials and methods: A retrospective study was carried out to evaluate sensitivity, specificity, positive predictive value (PPV), negative predictive value (NPV), concordance and sensitivity limits according to parasitemia ranges for the SD Bioline Malaria Antigen Pf/Pv ${ }^{\circledR}$ test in Cordoba and Choco. The results were compared with microscopy corrected by PCR.

Results: A total of 383 samples processed, 121 were positive (75 for $P$. vivax, 42 for $P$. falciparum and 4 for mixed infection) and 262 negative samples. $P$. vivax: sensitivity $92.0 \%$ (95\% Cl: 83.6-96.3), specificity 98.7\% ( 95\% Cl: 96.7-99.5), PPV 94.5\% (95\% Cl: 86.7-97.9), NPV 98.1\% (95\% Cl: 95.899.1), Cohen's kappa coefficient was 0.90 (0.80-1.00). P. falciparum: sensitivity $88.1 \%(95 \% \mathrm{Cl}: 75.0-$ 94.8), specificity 97.9\% (95\% Cl: 95.8-99.0), PPV 84.1\% (95\% Cl: 70.6-92.1), NPV 98.5\% (95\% IC:

\section{Contribución de los autores:}

Nohora Marcela Mendoza: diseño, coordinación del estudio, control de calidad, digitación de bases de datos, análisis de resultados y escritura del artículo.

Zulma Milena Cucunubá: diseño, análisis estadístico y escritura del artículo.

Samanta Aponte: diagnóstico molecular, análisis de resultados y escritura del artículo.

Nohora Elizabeth González: recolección y procesamiento de gota gruesa y prueba de diagnóstico rápido, análisis de resultados.

Sindy Durley Bernal: procesamiento de muestras para PCR y análisis de resultados. 
96.6-99.4), Cohen's kappa coefficient 0.80 (95\% Cl: 0.70-0.90).

Conclusions: The test performed well, being better for $P$. vivax as compared to $P$. falciparum. There are still difficulties of RDT to detect low parasitemias.

The non amplification of Pfhrp2 and Pfhrp3 genes in two samples diagnosed as mixed infection, suggest a possible deletion of these two genes together.

Key words: Malaria/diagnosis, evaluation studies, Colombia.

doi: http://dx.doi.org/10.7705/biomedica.v33i4.1464

Como línea de trabajo dentro de la estrategia mundial para la prevención y el control de malaria, se encuentra el diagnóstico temprano y el tratamiento oportuno y efectivo que son la base del manejo de la malaria y esenciales para reducir la morbimortalidad de la enfermedad. En el área del diagnóstico parasitario se han hecho desarrollos a nivel mundial que permiten tener en el mercado pruebas de diagnóstico rápido bien calificadas (1).

El diagnóstico parasitario de paludismo se hace con el fin de confirmar un caso, y orientar el tipo de medicamento y la atención que debe darse al paciente (2). La presencia del parásito en la sangre detectada mediante la gota gruesa, sigue siendo el diagnóstico de referencia por sus características técnicas, pues permite observar el parásito y logra brindar información sobre las características morfológicas y el recuento parasitario (3-5).

En Colombia, la malaria sigue siendo un problema de salud pública del que se reportó en el año 2011 un total de 65.172 casos, de acuerdo con la información del Sistema de Vigilancia de Salud Pública (6). Una de las causas por las cuales se logra mantener la transmisión del parásito y consecuentemente la ocurrencia del número de casos, es la falta de acceso del paciente a un diagnóstico oportuno en las zonas rurales, debido a que la atención en salud en Colombia se encuentra debilitada en estas áreas. Es por esto que, con el propósito de ampliar y fortalecer la red de diagnóstico en el país, se ha establecido como lineamiento desde el nivel nacional ampliar la cobertura y mejorar la oportunidad del diagnóstico por medio de las pruebas de diagnóstico rápido en aquellos lugares o circunstancias donde el diagnóstico microscópico no es viable, para lo cual se han destinado recursos económicos y se han

\section{Correspondencia:}

Nohora Marcela Mendoza, Laboratorio de Parasitología, Instituto Nacional de Salud, Avenida-calle 26 A № 51-20, Bogotá, D.C., Colombia

Teléfono: 220 7700, extensión 1337; teléfono celular: (300) 374 7820

marcemendoza07@gmail.com

Recibido: 16/11/12; aceptado:12/06/13 capacitado trabajadores de la salud y personas de la comunidad en el diagnóstico y tratamiento del paludismo (7).

Es posible adquirir diversas pruebas para el diagnóstico rápido de malaria, entre las que se encuentra la inmunocromatográfica SD Bioline Malaria Antigen $\mathrm{Pf} / \mathrm{Pv}{ }^{\circledR}$, la cual tiene una presentación en dispositivo (casete). En esta prueba se usa un anticuerpo monoclonal específico contra la proteína II rica en histidina (HRP-II) de Plasmodium falciparum y otro anticuerpo monoclonal específico para la detección de la lactato deshidrogenasa de $P$. vivax, haciendo posible diagnosticar infecciones causadas por $P$. falciparum o $P$. vivax de manera independiente 0 en infecciones mixtas por estas dos especies con un patrón de lectura diferente que, para efectos del país, resulta ser muy útil debido a la alta prevalencia de estas dos especies en Colombia (6).

Además, la prueba SD Bioline Antigen Pf/Pv ${ }^{\circledR}$ fue diseñada para ser almacenada y trabajada a temperaturas de 1 a $40^{\circ} \mathrm{C}$, lo cual la hace aplicable en la mayoría del territorio tropical sin requerir temperatura de refrigeración.

Debido a que dentro de la estrategia de fortalecimiento de la red de diagnóstico de malaria, el país adquirió esta prueba de diagnóstico rápido para aplicarla en cinco departamentos endémicos, fue necesario que el Instituto Nacional de Salud, como ente rector de la Red Nacional de Malaria en Colombia, evaluara las características operativas (sensibilidad, especificidad, valor diagnóstico positivo y negativo), así como su concordancia y límites de sensibilidad por rangos de parasitemia, en los departamentos de Córdoba y Chocó. Los resultados fueron comparados con la prueba de referencia que, en este caso, fue la gota gruesa corregida por la reacción en cadena de la polimerasa (PCR).

\section{Materiales y métodos}

\section{Tipo de estudio}

Se llevó a cabo un estudio retrospectivo para evaluar la precisión diagnóstica de la prueba SD 
Bioline Antigen $\mathrm{Pf} / \mathrm{Pv}$ ( paludismo en zonas endémicas. Se determinaron las características intrínsecas (sensibilidad y especificidad) y extrínsecas (VPP y VPN), así como la concordancia con el índice kappa (IK) de dicha prueba de diagnóstico rápido frente a la prueba diagnóstica de referencia.

\section{Diagnóstico de referencia}

Todas las muestras se analizaron a ciegas por microscopía y PCR. Como diagnóstico de referencia se utilizó el resultado de la gota gruesa corregida por la PCR. En el caso de discordancia entre los resultados de microscopía y PCR, se tomó el resultado de la PCR, excepto en aquellos en que la gota gruesa evidenció formas parasitarias y la PCR fue negativa.

La PCR utilizada ha demostrado alta sensibilidad y amplifica ADN parasitario viable o durante las primeras 24 horas después de muertos los parásitos, lo cual permite hacer diagnósticos frente a verdaderas infecciones parasitarias y no frente al ADN circulante derivado de parásitos muertos después de 24 horas (8).

\section{Lugar y periodo de estudio}

Las zonas fueron seleccionadas por tener un alto reporte de casos de malaria en Colombia. Para el año 2009, se reportó en Colombia un total de 79.198 casos de malaria por $P$.vivaxy $P$. falciparum, de los cuales el departamento del Chocó aportó el $6,6 \%$ y, el departamento de Córdoba, el 13,1\%, mientras que para el año 2010 , se reportó un total de 116.555 casos de los cuales Chocó aportó el $19 \%$ y Córdoba el $17 \%(9,10)$.

El estudio se realizó en tres puestos de diagnóstico microscópico que contaban con alta afluencia de pacientes. Dos puestos se encontraron ubicados, uno en Tierralta y otro en Puerto Libertador, dos municipios que pertenecen al departamento de Córdoba. El tercer puesto se localizó en el municipio de Quibdó en el departamento de Chocó. La captación de pacientes se hizo del 16 de noviembre al 2 de diciembre de 2010 en Córdoba y del 14 al 25 al junio de 2011 en el Chocó.

\section{Población de pacientes}

El estudio se llevó a cabo en todas las personas que asistieron a los puestos de microscopía en búsqueda de un diagnóstico de malaria y que cumplieron con la definición de caso probable de malaria planteada para este estudio.

\section{Caso probable}

Se definió como el paciente con fiebre actual o reciente (hasta 72 horas), que procedía de área endémica en los últimos 15 días y que pudiera tener o no relación epidemiológica con casos diagnosticados.

\section{Criterios de inclusión}

Se incluyeron pacientes que cumplieran con la definición de caso probable con edad igual o mayor de 6 años y que perteneciera o no al sistema de salud.

Se incluyeron solamente los pacientes que tuvieron un adecuado diligenciamiento del consentimiento informado o asentimiento en caso de menores de edad.

\section{Criterios de exclusión}

Se excluyeron en caso de retiro del consentimiento informado o asentimiento en caso de menores de edad; falta de diligenciamiento de la ficha clínicoepidemiológica, o presencia de signos o síntomas de malaria complicada.

\section{Tamaño de la muestra}

El tipo de muestreo utilizado fue de método no probabilístico, por conveniencia, es decir, se incluyeron todos los pacientes que acudieron al puesto de diagnóstico de malaria y que cumplieron los criterios de inclusión, hasta completar el número necesario de muestras.

Con base en la tendencia de casos en estas dos zonas endémicas, se asumió una probabilidad promedio de resultado positivo de 20 (comunicaciones personales de los grupos de Epidemiología de la Secretaría de Desarrollo de la Salud de Córdoba y el Departamento Administrativo de Salud de Chocó, bases de datos de notificación de muestras de malaria de los periodos epidemiológicos del 1 al 11 de 2010, suministradas el 8 de noviembre de 2010).

Además, según los resultados de evaluaciones previas hechas por la Organización Mundial de la Salud (OMS), se asumió que esta prueba contaba con una sensibilidad y especificidad del $95 \%$, un poder del $80 \%$ y una precisión del $5 \%$ (11). De esta manera, se estimó como mínimo un tamaño de muestra de 366 pacientes.

\section{Procedimiento en campo}

A los pacientes que acudieron a los puestos de diagnóstico de malaria se les brindó la atención rutinaria, siguiendo los protocolos de diagnóstico y tratamiento orientados por el nivel central y 
departamental, labor que estuvo a cargo del personal del puesto de diagnóstico. En cada puesto se informó el objetivo del estudio a todos los que acudieron en busca de atención y con aquellos que accedieron a participar se procedió de la siguiente manera:

1. Se aplicaró la encuesta clínico-epidemiológica y se obtuvo el consentimiento o asentimiento informado.

2. A todo paciente que cumplió con la definición de caso y los criterios de inclusión, se le tomaron muestras en dos láminas para gota gruesa y dos tubos de $4,5 \mathrm{ml}$ de sangre anticoagulada con EDTA. De los dos tubos, el primero se utilizó para procesar la prueba de diagnóstico rápido y, el segundo, para impregnar el papel filtro Whatman № 3 , con 8 pozos de $1,5 \mathrm{~cm}$ de diámetro, con el que se procesaron las pruebas de PCR.

Las gotas gruesas fueron tomadas y procesadas siguiendo las normas técnicas nacionales para el diagnóstico de malaria (12). La primera gota gruesa se utilizó para el diagnóstico y recuento parasitario en los puestos de microscopía; la segunda lámina se utilizó para hacer el control de calidad del diagnóstico por un segundo lector experimentado del Instituto Nacional de Salud. Cuando hubo discordancia entre el primer y el segundo lector, se procedió a hacer la lectura de la gota gruesa por un tercer lector. Todos los lectores realizaron el diagnóstico en ciego.

Las muestras de gotas gruesas se consideraron negativas cuando no se detectaron parásitos en toda la muestra observada.

La densidad parasitaria se determinó en 100 leucocitos. Cuando el número de parásitos fue inferior a 10, el recuento se llevó a 200 leucocitos. Para hacer los cálculos de las parasitemias, se utilizó como valor de referencia 8.000 leucocitos/ $/$ l.

3. Las pruebas de diagnóstico rápido fueron procesadas e interpretadas siguiendo los lineamientos del fabricante. El lavado se hizo con cuatro gotas del diluyente, pero cuando la tirilla no se observaba limpia a los 15 minutos o no migraba la muestra de manera adecuada, se adicionaron dos gotas de diluyente. El tiempo de lectura nunca excedió los 30 minutos. Estas pruebas fueron tomadas y procesadas en el mismo puesto de diagnóstico por una persona debidamente entrenada y el resultado se mantuvo en reserva para no interferir con los resultados de la gota gruesa.

4. Para el diagnóstico mediante PCR, se tomaron muestras de sangre de alrededor de $100 \mu \mathrm{l}$ las cuales se depositaron sobre papel filtro Whatman № 3 y se dejaron secar a temperatura ambiente. El almacenamiento de las muestras se llevó a cabo en bolsas de cierre hermético individuales con una bolsa de sílica gel y se mantuvieron en un lugar fresco y a temperatura ambiente hasta su Ilegada al Instituto Nacional de Salud para su procesamiento. Las muestras fueron diagnosticadas en ciego (13-15).

\section{Procesamiento para la PCR}

El procesamiento para el diagnóstico molecular de las muestras recolectadas se hizo en el Laboratorio de Bioquímica y Biología Celular.

A partir de las muestras de sangre recolectadas en papel filtro, se extrajo medio círculo $(0,75 \mathrm{~cm})$ de papel filtro Whatman y se le adicionó saponina a una concentración de 0,5\% durante 24 horas, con el fin de obtener la lisis de los glóbulos rojos. Posteriormente, se hicieron varios lavados con PBS 1X para retirar los excesos de hemoglobina y luego el papel filtro se pasó a otro tubo que contenía $200 \mu$ de la resina de Chelex a una concentración del $5 \%$, la cual fue precalentada a $99{ }^{\circ} \mathrm{C}$. El sobrenadante recolectado que contenía los ácidos nucleicos después de la centrifugación a $13.000 \mathrm{rpm}$, se distribuyó en alícuotas de $50 \mu \mathrm{l}$ y se almacenó a $-70^{\circ} \mathrm{C}$.

Una vez extraído el ADN, se hizo el diagnóstico de género y especie de Plasmodium. Para esto se llevó a cabo una primera reacción en la que se emplearon los iniciadores específicos de género rPLU6 y rPLU5, los cuales amplifican una región conservada de la subunidad pequeña del ARN ribosómico presente en todas las especies de Plasmodium, según la metodología descrita por Snounou, et al. (16). Partiendo de este producto, se hizo una segunda amplificación con iniciadores para la determinación de especie (rFAL1, rFAL2, rVIV1, rVIV2, rMAL1, rMAL2), los cuales amplifican regiones específicas del ARN ribosómico de $P$. falciparum, $P$. vivax o $P$. malariae, según la metodología descrita por Singh $(16,17)$.

Para la PCR de amplificación de $P$. falciparum, se empleó como control positivo el ADN de la cepa de 
referencia 3D7. En ausencia de cepas de referencia para $P$. vivax y $P$. malariae, se empleó ADN proveniente de pacientes tipificados previamente. En el caso de $P$. vivax, se empleó como control positivo el ADN de un aislamiento previamente tipificado con diferentes marcadores moleculares para $P$. vivax. El control positivo que se empleó para la amplificación de $P$. malariae fue donado por la Universidad Central de Venezuela, el cual fue extraído de un paciente con malaria mixta por $P$. falciparum y $P$. malariae. Asimismo, para cada una de las reacciones se incluyeron controles sin ADN y controles de la extracción obtenidos a partir de papel filtro sin ADN.

Los productos obtenidos en la PCR anidada se sometieron a electroforesis en gel de agarosa al $2 \%$ y se tiñeron con bromuro de etidio $(1 \mu \mathrm{g} / \mathrm{ml})(16)$.

La visualización de las bandas se hizo bajo luz ultravioleta, empleando el documentador de imágenes BIO-RAD Gel DocTM XR y el software One DScan, versión 3.1.

\section{Análisis estadístico}

Los datos se tabularon en MS Excel 8.0. La información se analizó con el software Stata versión 10.0. Se estimaron la sensibilidad, la especificidad, los valores predictivos, el índice kappa y la razón de verosimilitud en total y por rangos de parasitemias para tres categorías: $P$. vivax, $P$. falciparum e infección mixta. Los rangos de parasitemia $(<500$, $500-5.000$ y $>5.000$ parásitos/ $\mu$ l) se establecieron de acuerdo con la distribución de los datos en la muestra, para lograr grupos equitativos de análisis para las tres categorías.

Las frecuencias se expresaron en porcentaje para las variables cualitativas y como promedios para las cuantitativas. La prueba de ji al cuadrado se utilizó para determinar la significancia estadística y, en caso de pequeños tamaños de muestra, se utilizó la prueba exacta de Fisher. Un valor de $p<0,05$ se consideró como estadísticamente significativo.

Para las estimaciones por especie, se elaboró la tabla de contingencia, teniendo presente las muestras positivas para la especie frente al total de muestras negativas para dicha especie y se elaboró un cálculo independiente para los casos de infección mixta.

\section{Aspectos éticos}

El presente trabajo fue sometido a estudio y aprobación por el Comité de Ética del Instituto Nacional de Salud; el Acta $N^{\circ} 10$ del 31 de agosto de 2010, registra que se están cumpliendo estrictamente con lo estipulado en la Resolución No. 008430 de 1993 del Ministerio de Salud, por la cual se fijaron las normas éticas para la investigación en salud. De acuerdo con esta resolución, el estudio fue considerado como de riesgo mínimo, pues se obtuvieron dos muestras de sangre venosa de $4,5 \mathrm{ml}$ tomada por personal experto. Se explicaron a los pacientes el propósito del estudio, y los beneficios y riesgos de participar en él. Aquellos que aceptaron voluntariamente participar en el trabajo, firmaron el formato de consentimiento o asentimiento informado y fueron atendidos en el puesto de diagnóstico siguiendo la conducta de rutina. El diagnóstico se basó en el examen de la gota gruesa y los casos positivos se trataron de acuerdo con los lineamientos nacionales vigentes (2).

\section{Resultados}

\section{Línea de base demográfica y epidemiológica}

De las 383 muestras incluidas, 233 (60,8 \%) procedían de Córdoba, de las cuales, 121 (31,6 $\%)$ eran de Tierralta y $112(29,2 \%)$ de Puerto Libertador; las 150 (39,2\%) restantes se tomaron en el departamento de Chocó, en el municipio de Quibdó.

De los 383 pacientes estudiados para malaria, 201 $(52,5 \%)$ eran hombres y $182(47,5 \%)$ eran mujeres.

De los 383 pacientes, 93 (24,3\%) se encontraron en el rango de edad entre 6 y 16 años, 100 (26,1\%), entre 17 y 27 años, 100 (26,1\%) entre 28 y 42 años, y 90 (23,5\%), entre 43 y 92 años.

La frecuencia de los síntomas fue la siguiente: fiebre, 383 (100\%), cefalea, 333 (86,9\%), escalofríos, 257 (67,1\%), sudoración, 157 (41,0\%), ostealgia, 129 (33,7\%), mialgias, $111(29,0 \%)$, diarrea, $34(8,9 \%)$, y hemorragias, $4(1,0 \%)$.

Del total de pacientes, 30 (7,8 \%) recibieron tratamiento en el mes previo a la consulta para tratar un episodio de malaria.

\section{Desempeño de la prueba de diagnóstico rápido en comparación con el diagnóstico de referencia}

Los resultados para las cuatro categorías ( $P$. vivax, $P$. falciparum, infección mixta y negativas), comparando los diagnósticos obtenidos mediante la prueba de diagnóstico rápido frente a los obtenidos mediante la gota gruesa corregida por PCR, se muestran en el cuadro 1. 
La sensibilidad, especificidad, VPP, VPN, índice kappa y razón de verosimilitud por especie, se muestran en el cuadro 2. Además, la sensibilidad y especificidad por rango de parasitemia para $P$. vivax se muestra en el cuadro 3 y, para $P$. falciparum, en el cuadro 4. La parasitemia para $P$. vivax osciló entre 40 y 40.000 parásitos $/ \mu l$ y para $P$. falciparum osciló entre 32 y 23.726 formas asexuadas/ $\mu$ l.

En la figura 1 se observa la distribución de las muestras con bajas parasitemias ( $<500$ parásitos/ $\mu l)$ para $P$. vivax y $P$. falciparum.

Los resultados de las muestras en las cuales la prueba de diagnóstico rápido tuvo un resultado falso negativo con respecto a uno o a los otros dos métodos utilizados, la gota gruesa o la PCR, se muestran en el cuadro 5.

Para el caso de las cuatro muestras con infección mixta, la prueba de diagnóstico rápido, por sí sola, detectó $P$. vivax en todos los casos. Dos de estas tenían $P$. falciparumen cantidades submicroscópicas y las otras dos contaban con una parasitemia de 360 y 2.160 trofozoítos/ $\mu$ le $P$. falciparum.

A estas dos últimas muestras se les practicó amplificación del exón 2 de los genes Pfhrp2 y Pfhrp3, como ensayo preliminar, utilizando la metodología descrita por Backer, et al., en el año 2005 (18), evidenciándose ausencia de la amplificación de los genes en las dos muestras evaluadas (dato no mostrado).

En las tres muestras con gametocitemia pura para $P$. falciparum, la prueba de diagnóstico rápido no detectó una muestra con 40 gametocitos/ $\mu$ l, la cual presentaba la morfología destruida.

\section{Discusión}

En el presente estudio se evaluó la precisión diagnóstica de la prueba de diagnóstico rápido SD

Cuadro 1. Comparación del diagnóstico de malaria por especie mediante prueba de diagnóstico rápido frente a la gota gruesa corregida por PCR

\begin{tabular}{lccrrr}
\hline PDR & \multicolumn{5}{c}{ Gota gruesa corregida por PCR } \\
\cline { 2 - 5 } & $\begin{array}{c}\text { Plasmodium } \\
\text { vivax }\end{array}$ & $\begin{array}{c}\text { Plasmodium } \\
\text { falciparum }\end{array}$ & $\begin{array}{c}\text { Infección } \\
\text { mixta }\end{array}$ & Negativo & Total \\
\hline Plasmodium vivax & 69 & 0 & 4 & 073 \\
Plasmodium falciparum & 0 & 37 & 0 & 744 & 0 \\
Infección mixta & 0 & 0 & 0 & 255 & 266 \\
Negativo & 6 & 5 & 0 & 262 & 383 \\
Total & 75 & 42 & 4 & \\
\hline
\end{tabular}

PDR: prueba de diagnóstico rápido SD Bioline Malaria Antigen $\mathrm{Pf} / \mathrm{Pv}{ }^{\circledR}$

PCR: reacción en cadena de la polimerasa

Cuadro 2. Características operativas de la prueba rápida SD Bioline Malaria Antigen $\mathrm{Pf} / \mathrm{Pv}{ }^{\circledR}$ por especies

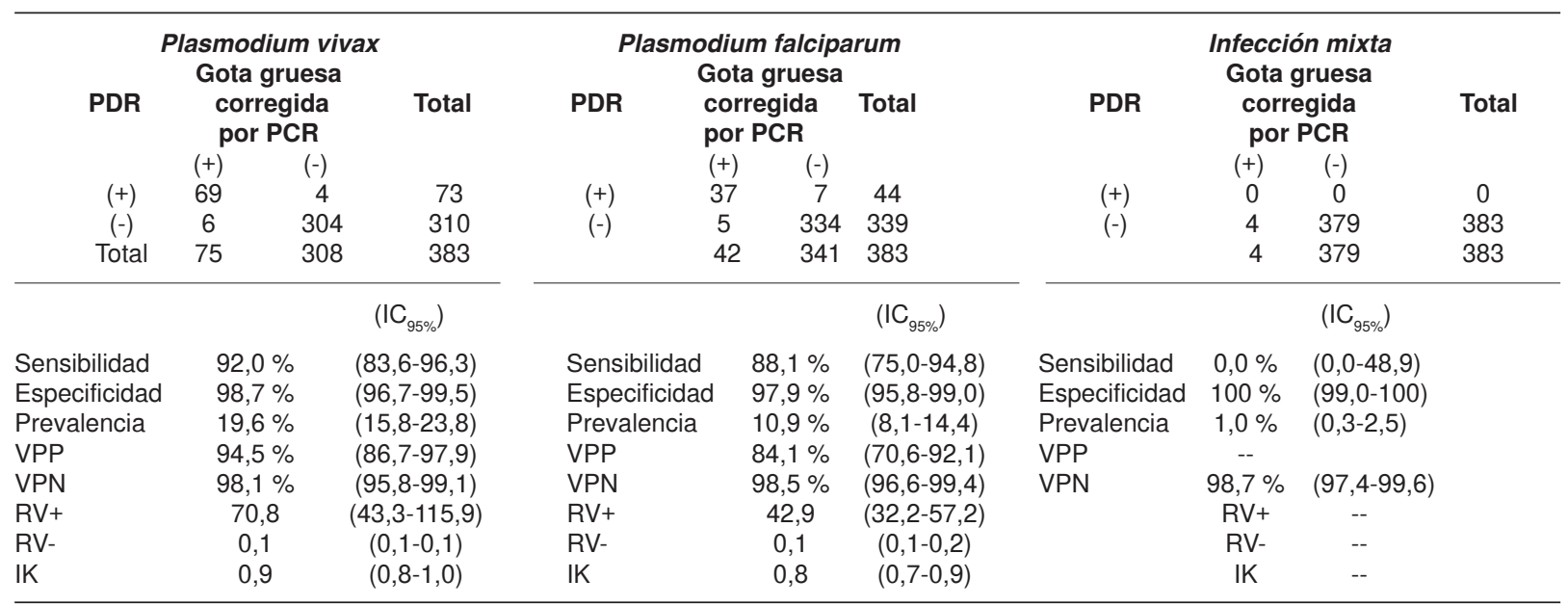

VPP: valor diagnóstico positivo; VPN: valor diagnóstico negativo; RV+: razón de verosimilitud positiva (likelihood ratio); RV-: razón de verosimilitud negativa; IK: índice kappa de Cohen.

PDR: prueba de diagnóstico rápido SD Bioline Malaria Antigen Pf/Pv®; PCR: reacción en cadena de la polimerasa; IC ${ }_{95 \%}$ : intervalo de confianza del $95 \%$ 
Cuadro 3. Sensibilidad y especificidad de la prueba de diagnóstico rápido SD Bioline Malaria Antigen Pf/Pv $®$ por rango de parasitemia para Plasmodium vivax

\begin{tabular}{|c|c|c|c|c|c|c|}
\hline \multirow[b]{2}{*}{$\begin{array}{l}\text { Rango } \\
\text { (parásitos/ } \mu \mathrm{l} \text { ) }\end{array}$} & \multicolumn{6}{|c|}{ Plasmodium vivax } \\
\hline & PDR & \multicolumn{2}{|c|}{$\begin{array}{c}\text { Gota gruesa corregida } \\
\text { por PCR }\end{array}$} & Total & $\begin{array}{l}\text { Sensibilidad } \\
\quad\left(\mathrm{IC}_{95 \%}\right)\end{array}$ & $\begin{array}{l}\text { Especificidad } \\
\quad\left(\mathrm{IC}_{95 \%}\right)\end{array}$ \\
\hline \multirow[t]{3}{*}{$<500$} & $(+)$ & 8 & 0 & 8 & $57,14 \%$ & \\
\hline & $(-)$ & 6 & 12 & 18 & $(32,6-78,6)$ & \\
\hline & Total & 14 & 12 & 26 & & \\
\hline \multirow[t]{3}{*}{$500-5.000$} & $(+)$ & 37 & 0 & 37 & $100 \%$ & \\
\hline & $(-)$ & 0 & 11 & 11 & $(90,8-100)$ & \\
\hline & Total & 37 & 11 & 48 & & \\
\hline \multirow[t]{3}{*}{$>5.000$} & $(+)$ & 24 & 0 & 24 & $100 \%$ & \\
\hline & $(-)$ & 0 & 16 & 16 & $(86,7-100)$ & \\
\hline & Total & 24 & 16 & 40 & & \\
\hline \multirow[t]{3}{*}{ No $P$. vivax } & $(+)$ & 0 & 4 & 4 & & $98,51 \%$ \\
\hline & $(-)$ & 0 & 265 & 265 & & $(96,24-99,42)$ \\
\hline & Total & 0 & 269 & 269 & & \\
\hline Total & & 75 & 308 & 383 & & \\
\hline
\end{tabular}

PDR: prueba de diagnóstico rápido SD Bioline Malaria Antigen Pf/Pv®

PCR: reacción en cadena de la polimerasa

Cuadro 4. Sensibilidad y especificidad de la prueba de diagnóstico rápido SD Bioline Malaria Antigen Pf/Pv ® por rango de parasitemia para Plasmodium falciparum

\begin{tabular}{|c|c|c|c|c|c|c|}
\hline \multirow[b]{2}{*}{$\begin{array}{l}\text { Rango } \\
\text { (parásitos/ } \mu \mathrm{l} \text { ) }\end{array}$} & \multicolumn{5}{|c|}{ Plasmodium falciparum } & \multirow[b]{2}{*}{$\begin{array}{l}\text { Especificidad } \\
\qquad\left(\mathrm{IC}_{95 \%}\right)\end{array}$} \\
\hline & PDR & $\begin{array}{r}\text { Gota gr } \\
(+)\end{array}$ & $\begin{array}{l}\text { corregida } \\
\text { CR } \\
(-)\end{array}$ & Total & $\begin{array}{l}\text { Sensibilidad } \\
\quad\left(\mathrm{IC}_{95 \%}\right)\end{array}$ & \\
\hline$<500$ & $\begin{array}{c}(+) \\
(-) \\
\text { Total }\end{array}$ & $\begin{array}{r}8 \\
4 \\
12\end{array}$ & $\begin{array}{r}0 \\
14 \\
14\end{array}$ & $\begin{array}{r}8 \\
18 \\
26\end{array}$ & $\begin{array}{c}66,67 \% \\
(39,1-86,2)\end{array}$ & \\
\hline $500-5000$ & $\begin{array}{c}(+) \\
(-) \\
\text { Total }\end{array}$ & $\begin{array}{r}11 \\
0 \\
11\end{array}$ & $\begin{array}{r}0 \\
37 \\
37\end{array}$ & $\begin{array}{l}11 \\
37 \\
48\end{array}$ & $\begin{array}{c}100 \% \\
(74,1-100)\end{array}$ & \\
\hline$>5.000$ & $\begin{array}{c}(+) \\
(-) \\
\text { Total }\end{array}$ & $\begin{array}{r}16 \\
0 \\
16\end{array}$ & $\begin{array}{r}0 \\
24 \\
24\end{array}$ & $\begin{array}{l}16 \\
24 \\
40\end{array}$ & $\begin{array}{c}100 \% \\
(80,7-100)\end{array}$ & \\
\hline No $P$. falciparum & $\begin{array}{c}(+) \\
(-) \\
\text { Total }\end{array}$ & $\begin{array}{r}2 \\
1 \\
3 \\
42\end{array}$ & $\begin{array}{r}7 \\
259 \\
266 \\
341\end{array}$ & $\begin{array}{r}9 \\
260 \\
269 \\
383\end{array}$ & & $\begin{array}{c}97 \% \\
(94,7-98,7)\end{array}$ \\
\hline
\end{tabular}

PDR: prueba de diagnóstico rápido SD Bioline Malaria Antigen Pf/Pv®

PCR: reacción en cadena de la polimerasa

Bioline Malaria Antigen $\AA^{\circledR} \mathrm{Pf} / \mathrm{Pv}$ por primera vez en Colombia, en pacientes sintomáticos en dos departamentos endémicos para malaria, uno con mayor prevalencia de $P$. falciparum (Chocó) y el otro con mayor prevalencia de $P$. vivax (Córdoba). Los resultados muestran una buena precisión de la prueba debido a que las muestras no detectadas contaban con parasitemias inferiores a 120 parásitos/ $\mu$ l, excepto en dos muestras con malaria mixta cuya parasitemia para $P$. falciparum fue de más de 200 parásitos/ $\mu \mathrm{l}$, de lo cual se deriva que estas pruebas permiten hacer diagnóstico de malaria con buena calidad. Estos resultados resultan relevantes en el contexto colombiano, pues en varios estudios se ha planteado que el uso adecuado de las pruebas de diagnóstico rápido puede mejorar el acceso y brindar mayor oportunidad en la atención en las poblaciones que habitan lugares rurales remotos en donde no se tiene acceso al diagnóstico, por la falta de cobertura de la red de diagnóstico microscópico, lo cual finalmente permite hacer uso racional del medicamento antipalúdico (19). Asimismo, el uso de este tipo de pruebas es cada vez más extendido 


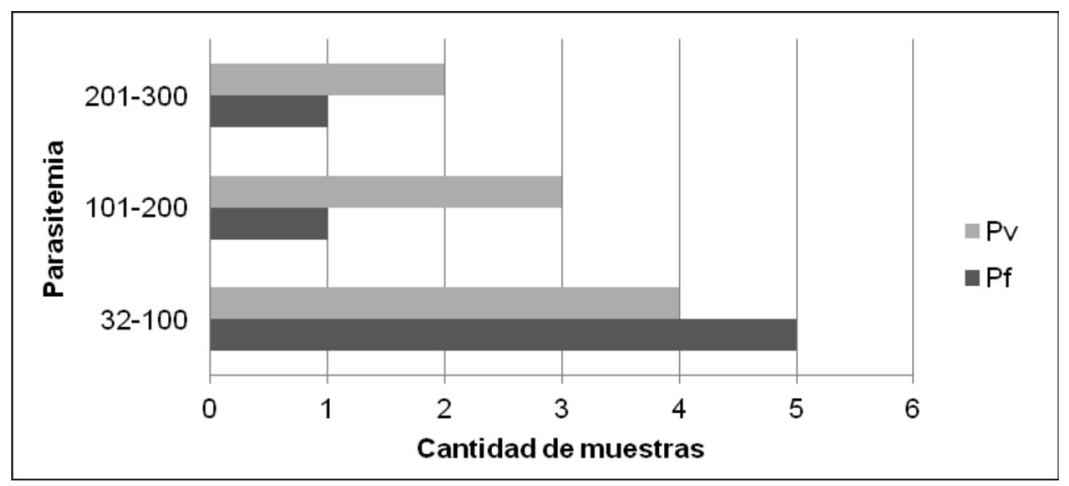

Figura 1. Distribución de muestras con bajas parasitemias para Plasmodium vivax y Plasmodium falciparum

Cuadro 5. Falsos negativos de la prueba de diagnóstico rápido SD Bioline Malaria Antigen Pf/Pv ${ }^{\circledR}$

\begin{tabular}{|c|c|c|c|c|c|c|}
\hline \multirow{2}{*}{$\begin{array}{l}\text { Muestra } \\
\text { Código }\end{array}$} & \multicolumn{3}{|c|}{ Gota gruesa } & \multirow[b]{2}{*}{ Estadio } & \multicolumn{2}{|c|}{ PCR } \\
\hline & Resultado & Especie & Parásitos/ $\mu \mathrm{l}$ & & Resultado & Especie \\
\hline 59 & + & $\mathrm{Pf}$ & 40 & Gametocitos* & $-\quad-$ & \\
\hline 244 & - & - & 0 & & $+\mathrm{Pf}$ & \\
\hline 248 & - & - & 0 & & $+\mathrm{Pf}$ & \\
\hline 172 & + & $\mathrm{Pf}$ & 80 & Trofozoítos & $+\mathrm{Pf}$ & \\
\hline 51 & + & $\mathrm{Pf}$ & 120 & Trofozoítos & $+\mathrm{Pf}$ & \\
\hline 44 & - & - & 0 & & $+\mathrm{PV}$ & \\
\hline 100 & - & - & 0 & & $+\mathrm{PV}$ & \\
\hline 255 & - & - & 0 & & $+P v$ & \\
\hline 87 & + & $\mathrm{Pv}$ & 32 & Todos & $+\mathrm{PV}$ & \\
\hline 243 & + & $\mathrm{Pv}$ & 40 & Todos & $+\mathrm{Pv}$ & \\
\hline 17 & + & $\mathrm{Pv}$ & 80 & Todos & $+\mathrm{PV}$ & \\
\hline
\end{tabular}

Cod: código, identificación; Pf: Plamodium falciparum; Pv: Plasmodium vivax; PDR: prueba de diagnóstico rápido; * destruidos; (-): negativo; (+) positivo.

debido a que se han implementado como una estrategia orientada por la OMS y financiada para apoyo de los programas de malaria en 81 países (20).

Los resultados encontrados para sensibilidad, especificidad, VPP e índice kappa para P. vivax, son mejores que para la detección de $P$. falciparum. No es posible comparar estos resultados con los de otros estudios que utilicen la misma prueba para el diagnóstico, debido a que no han sido realizadas en pacientes sintomáticos (21).

Las 11 muestras que tuvieron resultado falso negativo por la prueba de diagnóstico rápido al ser comparadas con la gota gruesa y la PCR, presentaban parasitemias de 120 o menos parásitos/ $\mu \mathrm{l}$, lo cual está dentro de los parámetros aceptados por la OMS, que consisten en que las pruebas de diagnóstico rápido deben detectar, como mínimo, parasitemias de 200 parásitos $/ \mu \mathrm{l}(22)$.

Por otra parte, de las tres muestras con gametocitemia pura de $P$. falciparum, solo en una no fue detectada por la prueba de diagnóstico rápido. Como posibles causas se tienen la baja parasitemia (40 gametocitos $/ \mu$ le sangre) y la morfología de los gametocitos que se encontraba destruida posiblemente porque eran formas muertas del parásito. Este último hecho daría una explicación más sólida del resultado negativo de la PCR. Además, es probable que los gametocitos encontrados en las muestras sean gametocitos maduros y, por tanto, no sean detectados por la prueba de diagnóstico rápido, ya que se ha demostrado que la proteína II rica en histidina ( HRP II ) es expresada por formas asexuadas y gametocitos inmaduros de $P$. falciparum y no por gametocitos maduros $(23,24)$.

Las pruebas de diagnóstico rápido que existen comercialmente se han centrado en la detección de la deshidrogenasa láctica parasitaria (pLDH), la HRP II y la aldolasa (23). Es así que en Colombia se han evaluado en los últimos años pruebas que detectan estos antígenos, como Now ICT Malaria Pf/ $\mathrm{Pv} \circledast$, Diamed OptiMAL-IT $\AA$ Malaria y Paracheck-Pf ${ }^{\circledR}$, con el fin de conocer su capacidad diagnóstica, generalmente con buenos resultados (25-28). 
Sin embargo, es importante seguir profundizando en la investigación de otros antígenos blanco con el fin de lograr niveles más altos en la sensibilidad y especificidad de las pruebas de diagnóstico rápido, y para evitar diagnósticos con resultados falsos negativos como ocurre en el caso de las cepas de $P$. falciparum que no expresan la HRP II, por deleción del gen que codifica para este antígeno, situación que se ha presentado principalmente en aislamientos de parásitos de la región amazónica $(14,29,30)$.

Las dos muestras en las cuales no se encontró amplificación de los genes Pfhrp2 y Pfhrp3, provenientes de Córdoba y que contaban con una parasitemia mayor de 200 parásitos $/ \mu \mathrm{l}$, mínima parasitemia exigida para la detección en las prueba de diagnóstico rápido, sugieren una probable deleción para estos genes en los dos aislamientos de $P$. falciparum. Sin embargo, es necesario confirmar estas deleciones empleando iniciadores para amplificación de genes corriente arriba y corriente abajo de Pfhrp2 y Pfhrp3, tanto en las dos muestras problema evaluadas en este trabajo como en otras muestras de la misma zona, con el fin de confirmar este hallazgo y poder dar recomendaciones técnicas que orienten las decisiones sobre la adquisición de pruebas de diagnóstico rápido ajustadas a las necesidades del país.

Si bien para la selección de esta prueba se tuvieron en cuenta los resultados de la evaluación de la Ronda 3 hecha a la prueba de diagnóstico rápido a través de la institución Foundation for Innovative New Diagnostics, y coordinada por la OMS, en la cual se presentaban valores de sensibilidad y especificidad más altos que los informados en el estudio actual, es importante tener en cuenta que estas evaluaciones se realizan bajo condiciones controladas de laboratorio, mientras que los resultados del presente trabajo están impactados por las condiciones de campo, variaciones ambientales, prevalencia de la enfermedad y el personal encargado de hacer los diagnósticos que difirió en los dos departamentos objeto (22).

En la literatura científica reciente se encuentran experiencias exitosas dirigidas a combatir la malaria en zonas de alto riesgo, como sucedió en Surinam, en donde actualmente se encuentran en fase de pre-eliminación de la malaria para lo cual integraron diferentes líneas de acción contra la enfermedad. Una de estas líneas fue la implementación del diagnóstico de paludismo mediante pruebas de diagnóstico rápido en áreas remotas sin diagnóstico microscópico, como en zonas mineras, en donde se desplazó personal entrenado en diagnóstico y tratamiento para proveer una atención gratuita (31). Por esto, se plantea que el uso extendido de las pruebas de diagnóstico rápido podría ser una herramienta crucial para aumentar el acceso y la oportunidad en zonas donde el diagnóstico microscópico es difícil de instaurar, como sucede en algunas zonas mineras de Colombia (32).

Finalmente, se plantea la necesidad de seguir fortaleciendo la incorporación de las pruebas rápidas en el Sistema de Gestión de la Calidad de la Red Nacional de Laboratorios, con el fin de vigilar y garantizar su correcta aplicación en situaciones de campo (33).

Como limitación del estudio se tiene el tamaño de muestra, debido a que este impacta la precisión de algunas estimaciones para $P$. falciparum y, especialmente para la infección mixta, lo cual genera intervalos de confianza amplios.

\section{Agradecimientos}

Los autores expresan sus agradecimientos a la Secretaría de Desarrollo de la Salud de Córdoba, al Departamento Administrativo de Salud de Chocó, a la Clínica Vida del Chocó por permitir la toma de muestra en los puestos de diagnóstico y proporcionar la información requerida para establecer el número de muestras del presente estudio. Además, a los grupos de Parasitología y Bioquímica del Instituto Nacional de Salud, por su apoyo en el procesamiento de las muestras, la lectura y el control de calidad.

\section{Financiación}

Este trabajo fue financiado con recursos de la Organización Panamericana de la Salud, Instituto Nacional de Salud, Fondo Mundial de la Lucha contra el Sida, la Tuberculosis y la Malaria subreceptor principal gobierno: Fondo Financiero de Proyectos de Desarrollo - FONADE a través del proyecto: "Uso de la inteligencia epidemiológica con participación social, para fortalecer la gestión del programa, mejorar el acceso al diagnóstico y tratamiento, y ejecutar intervenciones eficaces para la prevención y control de la malaria" y Annar Diagnostica Import, S.A.S.

\section{Conflictos de interés}

Los autores declaran que durante las fases de planeación, ejecución, evaluación, análisis de este trabajo, no incurrieron en conflictos de interés de ninguna naturaleza que hubieran podido afectar los resultados. 


\section{Referencias}

1. World Health Organization. Universal access to malaria diagnostic testing: An operational manual. Fecha de consulta: 24 de agosto de 2012. Disponible en: http:// www.who.int/malaria/publications/atoz/9789241502092/en/ index.html.

2. Instituto Nacional de Salud. Guía protocolo para la vigilancia en salud pública de malaria. Fecha de consulta: 24 de agosto de 2012. Disponible en: http://www.ins.gov.co/ temas-de-interes/Paginas/malaria.aspx.

3. Arróspide N, Flores R, Ruiz J. Evaluación de una prueba rápida para el diagnóstico de malaria en áreas endémicas del Perú. Rev Peru Med Exp Salud Pública. 2006;23:81-6.

4. Moody A. Rapid diagnostic tests for malaria parasites. Clin Microbiol Rev. 2002;15:66-78. http://dx.doi.org/10.1128/ CMR.15.1.66-78.2002

5. Makler MT, Palmer CJ, Age AL. A review of practical techniques for the diagnosis of malaria. Ann Trop Med Parasitol. 1998;92:419-33.

6. Instituto Nacional de Salud. Paludismo 2011. Informe del evento paludismo, hasta el periodo epidemiológico decimotercero del año 2011. Fecha de consulta: 22 de octubre de 2012. Disponible en: http://www.ins.gov. co/lineas-de-accion/Subdireccion-Vigilancia/Paginas/ informes-de-evento.aspx

7. Ospina C. Malaria cazada por campesinos microscopistas. El Mundo.com. Fecha de consulta: 18 de septiembre de 2012. Disponible en: http://www.elmundo.com/portal/ resultados/detalles/?idx=119188.

8. Jarra W, Snounou G. Only viable parasites are detected by PCR following clearance of rodent malarial infections by drug treatment or immune responses. Infect Immun. 1998;66:3783-7.

9. Instituto Nacional de Salud. Malaria 2009. Comportamiento de la malaria en Colombia según los casos notificados en SIVIGILA en 2009. Fecha de consulta: 22 de marzo de 2011. Disponible en: http://www.ins.gov.co/lineas-de-accion/ Subdireccion-Vigilancia/Paginas/informes-de-evento.aspx.

10. Instituto Nacional de Salud. Paludismo 2010. Informe del evento paludismo, 2010. Fecha de consulta: 22 de marzo de 2011. Disponible en: http://www.ins.gov.co/lineas-de-accion/ Subdireccion-Vigilancia/Paginas/informes-de-evento.aspx.

11. Foundation for Innovative New Diagnostics. Malaria RDT product testing: Interactive guide. Fecha de consulta: 20 de septiembre de 2012. Disponible en: http://www. finddiagnostics.org/programs/malaria-afs/malaria/rdt quality_control/product_testing/malaria-rdt-product-testing/ index.jsp.

12. Mendoza NM, Nicholls RS, Olano VA, Cortés LJ. Manejo integral de malaria. Santa Fe de Bogotá: Instituto Nacional de Salud; 2000.

13. Färnert A, Arez AP, Correia AT, Björkman A, Snounou G, do Rosário V. Sampling and storage of blood and the detection of malaria parasites by polymerase chain reaction. Trans R Soc Trop Med Hyg. 1999;93:50-3.

14. Gamboa D, Ho MF, Bendezu J, Torres K, Chiodini PL, Barnwell JW, et al. A large proportion of P. falciparum isolates in the Amazon region of Peru lack pfhrp2 and pfhrp3: Implications for malaria rapid diagnostic tests. PLoS Pathog. 2010;6:e1000830. http://dx.doi.org/10.1371/journal. ppat. 1000830 .

15. Baker J, Ho MF, Pelecanos A, Gatton M, Chen N, Abdullah $\mathrm{S}$, et al. Global sequence variation in the histidine-rich proteins 2 and 3 of Plasmodium falciparum: Implications for the performance of malaria rapid diagnostic tests. Malar $\mathrm{J}$. 2010;9:129. http://dx.doi.org/10.1186/1475-2875-9-129

16. Snounou G, Viriyakosol S, Zhu XP, Jarra W, Pinheiro L, do Rosario VE, et al. High sensitivity of detection of human malaria parasites by the use of nested polymerase chain reaction. Mol Biochem Parasitol 1993;61:315-20.

17. Singh B, Bobogare A, Cox-Singh J, Snounou G, Abdullah MS, Rahman AH. A genus and species-specific nested polymerase chain reaction malaria detection assay for epidemiologic studies. Am J Trop Med Hyg. 1999;60:68792.

18. Baker J, Mc Carthy J, Gatton M, Kyle DE, Belizario V, Luchavez J, et al. Genetic diversity of Plasmodium falciparum Histidine-Rich Protein 2 (PfHRP2) and its effect on the performance of PfHRP2-based rapid diagnostic tests. J Infect Dis. 2005;192:870-7. http://dx.doi. org/10.1086/432010

19. Masanja IM, Selemani M, Amuri B, Kajungu D, Khatib R, Kachur SP, et al. Increased use of malaria rapid diagnostic tests improves targeting of anti-malarial treatment in rural Tanzania: Implications for nationwide rollout of malaria rapid diagnostic tests. Malar J. 2012;11:221. http://dx.doi. org/10.1186/1475-2875-11-221.

20. Zhao J, Lama M, Korenromp E, Aylward P, Shargie E, Filler S, et al. Adoption of rapid diagnostic tests for the diagnosis of malaria, a preliminary analysis of the global fund program data, 2005 to 2010. PLoS One. 2012;7:e43549. http://dx.doi.org/10.1371/journal.pone.0043549

21. Jeremiah ZA, Uko EK, Buseri FI, Jeremiah TA. Field evaluation of SD Bioline rapid malaria diagnostic test among asymptomatic malaria infected children in Port Harcounrt, Nigeria. Res J Parasitol. 2007;2:39-44. http:// dx.doi.org/10.3923/jp.2007.39.44

22. World Health Organization. Malaria rapid diagnostic test performance. Results of WHO product testing of malaria RDTs: Round 3 (2010-2011). Geneva: WHO; 2011.

23. Pérez HA, Bracho C, De La Rosa M. El paludismo y las pruebas rápidas de diagnóstico. Bol Mal Salud Amb. 2007;47:3-13.

24. World Health Organization. Malaria diagnosis. New perspectives. WHO/MAL/200.1091. Geneva: WHO; 2000.

25. Ferro BE, González IJ, Carvajal F, Palma GI, Saravia NG. Performance of OptiMAL ${ }^{\circledR}$ in the diagnosis of Plasmodium vivax and Plasmodium falciparum infections in a malaria referral center in Colombia. Mem Inst Oswaldo Cruz. 2002;97:731-5. http://dx.doi.org/10.1590/S007402762002000500025

26. van den Broek I, Hill O, Gordillo F, Angarita B, Hamade $\mathbf{P}$, Counihan $\mathbf{H}$, et al. Evaluation of three rapid tests for diagnosis of $P$. falciparum and $P$. vivax malaria in Colombia. Am J Trop Med Hyg. 2006;75:1209-15.

27. Mendoza NM, García M, Cortés LJ, Vela C, Erazo R, Pérez $\mathrm{P}$, et al. Evaluación de dos pruebas rápidas [NOW® 
ICT Malaria Pf/Pv y OptiMAL®] para el diagnóstico de paludismo en Tumaco, Colombia. Biomédica. 2007;27:571 80.

28. Montoya AE, Menco J, Osorio N, Zuluaga MA, Duque $\mathrm{J}$, Torres $\mathrm{G}$, et al. Concordancia entre gota gruesa, inmunocromatografía y reacción en cadena de la polimerasa para el diagnóstico de malaria. Biomédica. 2008;28:252-61.

29. Maltha J, Gamboa D, Bendezu J, Sánchez L, Cnops L, Gillet $\mathrm{Ph}$, et al. Rapid diagnostic tests for malaria diagnosis in the Peruvian amazon: Impact of pfhrp2 gene deletions and cross-reactions. PLoS ONE. 2012;7:e43094. http:// dx.doi.org/10.1371/journal.pone.0043094

30. Houzé S, Hubert V, Le Pessec G, Le Bras J, Clain J. Combined deletions of pfhrp2 and pfhrp3 genes result in Plasmodium falciparum malaria false-negative rapid diagnostic test. J Clin Microbiol. 2011;49:2694-6. http:// dx.doi.org/10.1128/JCM.00281-11

31. Hiwat H, Hardjopawiro LS, Takken W, Villegas L. Novel strategies lead to pre-elimination of malaria in previously high-risk areas in Suriname, South America. Malaria J. 2012;11:10. http://dx.doi.org/10.1186/1475-2875-11-10.

32. Olano V, Carrasquilla G, Méndez F. Transmission of urban malaria in Buenaventura, Colombia: Entomological features. Rev Panam Salud Pública.1997;1:287-94.

33. Ospina OL, Cortés LJ, Cucunubá ZM, Mendoza NM, Chaparro P. Caracterización de la Red Nacional de Diagnóstico de Malaria, Colombia, 2006-2010. Biomédica. 2012;32:46-57. 